vol. 34 - $n^{\circ} 1 \mid 2018$

Mouvements migratoires d'hier et d'aujourd'hui en Italie

\title{
Racisme, antiracisme et migrations : l'Italie au cœur de la conjoncture politique européenne
}

Racism, Anti-racism and Migration: Italy at the Heart of the New European

Political Situation

Racismo, antirracismo y migración: Italia en el centro de la nueva situación

política europea

Miguel Mellino

Traducteur : Adelina Miranda

\section{(2) OpenEdition}

Journals

Édition électronique

URL : https://journals.openedition.org/remi/9630

DOI : $10.4000 /$ remi.9630

ISSN : $1777-5418$

Éditeur

Université de Poitiers

Édition imprimée

Date de publication : 1 avril 2018

Pagination : 11-19

ISBN : 979-10-90426-61-0

ISSN : 0765-0752

Référence électronique

Miguel Mellino, « Racisme, antiracisme et migrations : l'Italie au cœur de la conjoncture politique européenne », Revue européenne des migrations internationales [En ligne], vol. 34 - $n^{\circ} 1$ | 2018, mis en ligne le 28 décembre 2018, consulté le 19 avril 2022. URL : http://journals.openedition.org/remi/9630 DOl : https://doi.org/10.4000/remi.9630 


\title{
Chronique d'actualité
}

\section{Racisme, antiracisme et migrations : I'Italie au cour de la conjoncture politique européenne}

\author{
Miguel Mellino ${ }^{1}$
}

\section{Racisme à I'italienne}

L'actuelle amplification du phénomène de la violence raciste et des luttes antiracistes en Italie donnent vie à une dialectique qui est devenue évidente depuis l'arrivée au pouvoir d'une coalition qui se déclare ouvertement souverainiste et xénophobe ${ }^{2}$. Pour expliquer cette dialectique, deux éléments de contexte doivent être rappelés : l'accroissement du racisme dans ses expressions les plus violentes - institutionnelles ou non - qui a favorisé la victoire électorale du gouvernement actuel et la succession d'agressions et de faits racistes qui se déroulent sur le fond de la "crise des réfugiés" en lien avec le conflit syrien à partir de l'été 2015.

Dans cette série de faits exprimant la montée des actes racistes, rappelons en 2016, I'homicide du Nigérien Emmanuel Chidi Nnamdi à Fermo et celui de I'ouvrier égyptien Abd El Salam Ahmed El Danf à Plaisance alors qu'il participait à un piquet de grève; en 2017, la mort de la demandeuse d'asile ivoirienne Sandrine Bakayoko dans les toilettes du "centre d'accueil" de Conetta et le suicide du Gambien Pateh Sabally dans les eaux du Grand Canal de Venise non secouru par les touristes et les passants; la violente expulsion des migrants de la Place de I'Indépendance à Rome; en 2018, la mort de la Nigérienne Becky Moses à la suite de l'incendie des baraques du ghetto de Rosarno. Cette vague de violences racistes, loin d'être contenue ou réprimée, s'est au contraire accentuée, comme le démontrent des faits plus récents : à Macerata, en février 2018, des sympathisants néo-fascistes de Forza Nuova et de Casa Pound, deux forces politiques d'extrême droite, ont fusillé six migrants " noirs " en les blessant grièvement; en mars 2018, à Florence un retraité a tiré sans aucune motivation sur Idy Diene, une commerçante ambulante sénégalaise; en juin 2018, Soumaila Sacko, un activiste malien, engagé dans la lutte contre le système d'exploitation esclavagiste a été tué dans les campagnes calabraises.

1 Anthropologue et activiste au sein du mouvement antiraciste italien et européen. Professeur, Université de Naples "L'Orientale", Département des Sciences humaines et sociales, Via Chiatamone,61/62,80121 Naples, Italie; mamellino@unior.it La traduction de l'italien vers le français a été réalisée par Adelina Miranda.

2 Depuis juin 2018, I'Italie est gouvernée par une coalition politique composée par le Mouvement 5 Étoiles et la Ligue, un parti xénophobe et raciste. 
L'ensemble de ces morts violentes traduisent le changement qui s'opère dans le racisme en Italie et elles en disent probablement beaucoup plus sur les spectres qui agitent l'Europe en crise. Ces meurtres ont en partie été anticipés par l'affirmation de mouvements xénophobes qui les ont transformés en un instrument de consensus et de jonction nationale populaire. Par ailleurs, au cours des trois dernières années, ils ont fait l'objet d'une construction narrative politico-médiatique et d'une gestion institutionnelle tant de la part de I'Union européenne que du gouvernement italien précédent ${ }^{3}$.

C'est dans ce contexte qu'il faut situer la prise de position brutale de l'actuel gouvernement italien à l'encontre des migrants et des migrations. Le programme Immigration zéro (inspiré du célèbre No way du gouvernement australien), l'interdiction de porter secours aux migrants en mer ayant pour conséquence la criminalisation des ONG, I'approbation du décret sur la sécurité4, I'opposition farouche à tout dessein de loi favorisant le droit du sol (ius soli), l'abstention lors de la signature du Pacte mondial pour des migrations porté par l'Organisation des Nations unies, constituent un assemblage de mesures qui expriment les choix brutaux des forces politiques actuellement au pouvoir. Elles ont préféré sciemment s'appuyer sur les émotions qui traversent la société italienne depuis longtemps pour accéder au pouvoir et donner une nouvelle orientation à la politique à travers ce que je qualifie de "pacte raciste de citoyenneté", offert à une partie des citoyens au détriment des autres. De ce fait, sans minimiser les responsabilités du gouvernement actuel dans la "racialisation de la crise migratoire", ce serait une grave erreur de le considérer comme l'unique responsable de ce qui se déroule en Italie à propos des migrations et du racisme.

\section{L'Italie : un observatoire de la nouvelle conjoncture politique européenne}

Si l'on regarde l'affrontement entre le gouvernement italien et I'Union européenne à travers le prisme des migrations et du racisme, l'Italie peut être considérée comme étant un observatoire privilégié de la conjoncture politique européenne portée par de nouvelles formations politiques. Mon postulat est que le scénario transalpin nous renseigne sur la grammaire politique des "mouvements souverainistes" revendiquant un retour à une gestion nationale du territoire afin de se distinguer avec éclat de la dimension supranationale des institutions de I'Union européenne. Cette renationalisation de la souveraineté territoriale est constitutive de ce que je propose d'appeler la "crise de I'hégémonie".

L'affirmation du souverainisme en Europe, à la suite du triomphe de Donald Trump aux États-Unis, est un des symptômes de la "crise du consensus Maastricht-Schengen", c'est-à-dire de l'épuisement du modèle ordolibéral de gouvernance (notamment au niveau de la politique institutionnelle) promu par

\footnotetext{
3 Entre 2013 et 2018, un gouvernement de centre gauche a été au pouvoir. Au cours de ces cinq ans, trois Premiers ministres se sont succédé : Letta, Renzi et Gentiloni.

4 Ce décret, qui porte aussi le nom de "Décret Salvini ", a été approuvé le 29 novembre 2018. Il prévoit, entre autres, I'annulation des permis de séjour pour des raisons humanitaires et l'allongement de quatre-vingt-dix à 180 jours le temps "légal " de détention des migrants et des demandeurs d'asile dans les Hotspots.
} 
I'Union européenne au cours de ces vingt-cinq dernières années et sur lequel repose la construction de l'Union européenne. Cette "crise de l'hégémonie" débute en 2008 en raison des conséquences économiques du krach financier le plus important des dernières décennies qui a secoué l'équilibre institutionnel interne de I'Union européenne. La dureté des politiques d'austérité, l'action fiscale, les mesures déflationnistes couplées à la prolongation de la stagnation économique et à l'accroissement des conflits sociaux ont produit de fortes tensions entre certaines formations (notamment des catégories sociales et des régions moins liées au capital financier) et les institutions de I'Union européenne. Le coup de grâce au "consensus de Schengen" a été porté par la "crise des réfugiés" en 2015 avec l'émergence de redoutables mouvements de résistance qui ont fait exploser le management européen de la gestion des frontières mis en place au cours de ces vingt dernières années. Ce n'est donc pas un hasard si l'émergence du mouvement souverainiste comme alternative à la politique menée par I'Union européenne arrive dans le prolongement de la "crise des réfugiés". Crise économique et crise du régime migratoire ont marqué la fin du consensus ordolibéral de gouvernance européenne. Et encore une fois, le cas italien est à ce propos symptomatique.

Depuis 2015, I'ordolibéralisme et le souverainisme se sont définis comme deux projets permettant de "gouverner la crise". Toutefois, au-delà des différences réelles et importantes, ces deux programmes politiques ne sont pas étrangers l'un à l'autre. Mon objectif n'est donc pas de discuter les contrastes existant entre les deux formations, mais plutôt d'analyser les solutions politiques qu'ils proposent pour résoudre la crise et rétablir l'ordre européen. En effet, malgré les rhétoriques divergentes, caractérisées par deux types d'autoritarisme (I'un économico-fiscal, I'autre xénophobe) l'ordolibéralisme et le souverainisme présentent un air de famille. Ce qui les rapproche, voire les entremêle, est plus important que ce qui les différencie.

Dans les deux cas, le dispositif hégémonique du gouvernement porte sur un "nouveau pacte de citoyenneté" avec des milieux sociaux économiquement actifs et avec les classes populaires autochtones (blanches). Ce projet politique se fonde sur le contrôle toujours plus répressif de la population non européenne, notamment pour la partie qui peut potentiellement constituer une force de travail active sur le marché de l'emploi. La distribution sociale du "consensus" et de la "coercition" s'opèrent en fonction d'une persistante ligne de couleur. L'ordolibéralisme et le souverainisme sont deux solutions racistes à la crise qui puisent leur force politique et culturelle dans l'histoire coloniale et postcoloniale européenne, dans la fusion historique entre capitalisme et colonialisme (Hansen et Stepputat, 2005; Harvey, 2003 et 2005) et dans la construction matérielle de I'Europe de Maastricht.

De ce fait, il est difficile et politiquement inutile de considérer l'émergence du souverainisme en Europe comme étant externe au discours néo-ordolibéral européen. La construction narrative à la base de l'idéologie souverainiste se propose de restaurer un ordre économique, patriarcal et racial - considéré comme perdu - et de renouer avec le lien social du passé qui s'inscrivait dans la communauté et la tradition nationales et qui aurait été compromis par l'instabilité financière globale ainsi que par l'establishment politique national, et surtout celui européen. Comme le montre le cas italien, le mouvement souve- 
rainiste traduit ce "despotisme financier global » en une chaîne de significations perverse qui se révèle être profitable pour les marchés électoraux des sociétés européennes postcoloniales. II offre une décodification des effets déstabilisants du capitalisme financier global à travers une équation élaborée au niveau du discours sur la série synthétique suivante : Union européenne/flux migratoires/ criminalité/perte des postes de travail pour les autochtones.

Regardons le souverainisme européen au travers du prisme de la race, du racisme et des migrations, trois points à prendre en considération. En premier lieu, le souverainisme constitue désormais le pilastre d'un nouveau dispositif sécuritaire du contrôle social. Son énonciation politique représente une radicalisation de certaines tendances déjà inscrites dans le racisme institutionnel et structurel promu par le régime migratoire européen et qui ont constitué la base pour l'élaboration du dispositif néo-ordolibéral de production des territoires et des peuples. En deuxième lieu, les faits qui se déroulent depuis quelques mois en Italie démontrent que dans la gestion des migrations et des frontières en situation de post-crise il n'existe aucune césure significative entre les politiques de I'Union européenne et les propos du souverainisme. Cette convergence est confirmée par le fait que l'Union européenne ne s'est pas opposée au décret Sécurité voté par le gouvernement italien. En troisième lieu, la politique de celui-ci se base sur une gestion raciste de la crise migratoire pour relancer le système productif national à l'intérieur d'un modèle d'accumulation néolibéral valorisant l'identité, la propriété, la sécurité et la mise en concurrence. Le programme du gouvernement italien met en avant de petits correctifs en matière d'autonomie fiscale afin d'alléger les impositions émanant des politiques européennes, mais surtout certaines spécificités qui devraient défendre la "catégorie nationale" qui constitue une partie de son électorat. De ce fait, il repose sur la promesse d'une politique qui, sélective et policière, produit l'exclusion au nom du statut de citoyen.

Le souverainisme populiste européen reproduit la structure des émotions collectives utilisant ses insurmontables limites historiques : une conception blanche qui exclue et racialise le peuple. Ces limites s'enracinent dans I'histoire coloniale et impériale des États-nations modernes européens, dans la colonialité constitutive de la notion politico-culturelle du peuple produite disséminée dans les institutions, et dans le tissu colonial de leurs principales technologies du pouvoir et de l'assujettissement.

\section{La crise de I'antiracisme européen}

En Italie, la crise a activé une politisation du racisme suivie d'une racialisation de la sphère politique et des questions sociales. Néanmoins, il apparaît évident que les pratiques et les politiques antiracistes, même celles qui semblent être les plus radicales, sont dans une impasse. Et cela malgré les mobilisations métropolitaines antiracistes importantes (comme celle de Macerata, Florence, Milan et Rome) et les luttes des migrants pour revendiquer divers droits sociaux (logement, travail, nationalité, etc.) qui ont eu lieu au cours de deniers mois.

$C^{\prime}$ est une constatation essentielle sur laquelle il faut réfléchir. Et, encore une fois, I'Italie peut constituer un point d'observation paradigmatique : I'analyse sur 
les limites de l'antiracisme italien peut être élargie à d'autres contextes discursifs antiracistes européens. II me semble que dans ses expressions dominantes, l'antiracisme italien continue à se présenter sous des formes "éthico-morales" plutôt que politiques. Ce que je veux souligner c'est que l'antiracisme dominant n'est pas en mesure de se reformuler dans une conjoncture politique inédite s'il n'arrive pas à dépasser une vision critique de l'antiracisme de type «idéologicoculturel " (Fassin, 2017; Fassin et Fassin, 2012). Les conditions de vie de presque 6 millions d'immigrés résidant en Italie avec un permis de séjour régulier et l'expérience vécue par les secondes et les troisièmes générations révèlent l'existence d'une "racialisation" croissante aux niveaux économique et social. Or, I'antiracisme italien continue de lutter contre le racisme comme s'il dépendait exclusivement d'une sorte d' "identitarisme culturel ", c'est-à-dire d'une simple manipulation idéologique centrée sur un ensemble de représentations fausses, reliées à la discrimination, aux stigmatisations et à l'infériorisation.

En Italie, I'antiracisme est évoqué comme un moyen fondamental pour la recomposition politique seulement lorsque l'on veut distinguer les discours et les pratiques racistes des mouvements ouvertement xénophobes. L'antiracisme est ainsi mobilisé d'une manière autoréférentielle rapporté à un universalisme abstrait républicain et tant celui de gauche que de droite est incapable de rendre visible dans la sphère publique le fait que le racisme est un phénomène "historique" et "structurel» des sociétés européennes. Un des acquis épistémologiques les plus importants des études consacrées au racisme - des études classiques comme celles de Du Bois (1935), Fanon (1956) jusqu'aux plus récentes comme Hall (2015), Gilroy (1987), Roediger (2010), Goldberg (2015) et Taylor (2016) - est qu'il ne constitue pas un phénomène monolithique et identique partout. II se métamorphose constamment et est remodelé selon les contextes géohistoriques et les luttes antiracistes. Néanmoins, l'analyse de l'antiracisme dominante en Italie se réfère toujours à des textes des années 1980 et 1990, comme ceux de Taguieff (1988) et Wievorka (1991) ou encore à Race, nation et classe de Balibar et Wallerstein $(1988)^{5}$. Ces ouvrages ont eu le mérite de souligner l'importance du "racisme différentialiste" ou du "néo-racisme» promu par ceux qui à cette époque constituaient les mouvements s'opposant aux politiques migratoires européennes, parmi lesquels la Ligue lombarde qui est actuellement au gouvernement.

Toutefois, une des limites de ce modèle interprétatif est d'adopter l'antisémitisme nazi comme idéaltype du racisme moderne. Comme le souligne Balibar (2007), le migrant est le nom de la race, mais cette affirmation, certes importante, risque de réduire le racisme contemporain à un acte symbolique discriminatoire exercé à l'encontre de certains groupes. Cette posture produit une invisibilisation de la dimension historique et matérielle du phénomène, qui est articulée sur des dispositifs hiérarchisant (la racialisation) tous les citoyens, et de la colonialité, c'est-à-dire des conséquences du colonialisme sur la longue durée, conséquence du rapport historique de l'Europe avec les autres colonisés. Si nous voulons parler de racialisation, il faut considérer qu'elle ne peut pas être vue comme étant fausse et subjective, rapportée aux seules représentations privées ou à la simple sphère du "travail migrant". II s'agit d'un phénomène

5 Ce texte est sûrement plus complexe et articulé, mais il est établi sur des limites et des réductionnismes typiques du "marxisme blanc" pour affronter la question du racisme. 
qui traverse toute la population et dont les effets se matérialisent dans les différentes formations sociales à travers la distribution des hiérarchies et des privilèges.

De mon point de vue, la racialisation ne concerne donc pas qu'une partie de la société, les autres racisés. Ce phénomène permet de comprendre les effets matériels de l'intersection du capital avec les discours occidentaux produits sur la race, tant sur les espaces et les structures que sur les corps et les subjectivités. De conséquence, pour l'analyser il est nécessaire de considérer l'effet que la racialisation engendre sur le tissu social par une multiplicité de discours et de pratiques, institutionnels ou non, orientant la construction et la représentation hiérarchiques des différences ("physiques" et "culturels", "réelles " et "imaginaires") entre les groupes et les sujets ainsi que la manière de discipliner les rapports matériaux et intersubjectifs. En d'autres termes, le concept de racialisation, saturé de I'héritage colonial et impérial de la notion de "race", semble plus apte que d'autres concepts plus neutres (par exemple celui d'ethnicisation) pour décrire en mode efficace les processus d'essentialisation, infériorisation et ségrégation économique et culturelle, qui traduisent la violence matérielle et symbolique à laquelle sont actuellement soumis dans l'espace italien et européen les sujets appartenant à certains groupes (Mellino, 2012).

Le problème est qu'au niveau théorique l'analyse du racisme continue à se référer à la tradition du marxisme blanc européen sans utiliser l'enseignement de la tradition certainement plus riche et articulée des Black Studies et de la pensée radicale afro-américaine et afro-caribéenne (Gilroy, 1987; Mbembe, 2003; Roediger, 2010; Taylor, 2016). Ce n'est pas un hasard si en Italie des expressions comme "racisme structurel", "racisme institutionnel" ou "ségrégation sociale" ne sont pas utilisées et elles ne sont pas placées au cœur du débat antiraciste dominant. Celui-ci reste centré sur le principe de la solidarité et de l'identification politique (souvent paternaliste et victimisant) avec la cause des groupes et des sujets racisés plutôt que sur une pratique interprétative permettant la compréhension de la composition symbolique et matérielle des formations sociales contemporaines. C'est une faiblesse théorique doublement paradoxale. Tandis que le racisme se montre comme un dispositif de plus en plus au centre des processus de hiérarchisation constitutifs du néo-libéralisme, l'antiracisme - tout comme la gestion de la "crise migratoire" - apparaît comme un élément "accessoire" (Fanon, 1956) ou pour ainsi dire "externe" au programme politique et théorique porté par des sujets politiques non racistes. De plus, le débat critique sur les migrations est dominé par des paroles clefs comme "frontières", "intégration", "réfugiés" (Marchetti et Pinelli, 2016; Campesi, 2015; Mezzadra et Neilson, 2014) laissant un espace contingent et résiduel à la réflexion sur le rôle du racisme et de l'antiracisme dans l'actuelle construction tant de l'Italie que de l'Europe.

De ce fait, l'antiracisme non seulement arrive toujours en dernier dans le débat, mais il est souvent confiné au "sous-sol" de la lutte politique, dans un espace marginal et destiné à reconquérir une centralité temporaire - surtout de type morale - lorsque le cycle de violence réexplose dans des actes ou des discours. Et pour autant, une bonne partie des populations européennes (migrantes, post-migrantes, secondes générations, réfugiées, etc.) vit le racisme au quotidien comme un des dispositifs primaires de leur prolétarisation, 
d'inclusion différentielle et d'exclusion. Autrement dit, pour la majorité des Européens il est plutôt clair qu'en Europe la "production de la population" est historiquement articulé avec un dispositif racialisant de gouvernance qui prend ses origines dans le passé colonial et dans l'entremêlement entre capitalisme et colonialisme. Toutefois, en Europe on n'arrive pas à le concevoir comme étant quelque chose qui structure tant les conditions matérielles de la vie que les identités culturelles. C'est à partir de ces dernières considérations qu'il est nécessaire de repenser notre antiracisme pour l'avenir.

\section{Références bibliographiques}

Agier Michel (2014) Un monde de camps, Paris, La Découverte, 422 p.

Alexander Michelle (2012) The New Jim Crow: Mass Incarceration in the Age of Colorblindness, New York, The New press, $312 \mathrm{p}$.

Balibar Étienne (2007) Le retour de la race, Mouvements, 50, pp. 162-171.

Campesi Giuseppe (2016) Chiedere asilo in tempo di crisi : accoglienza, confinamento e detenzione ai margini d'Europa, in Chiara Marchetti e Barbara Pinelli Dirs., Confini d'Europa. Modelli di controllo e inclusioni formali, Milano, Raffaello Cortina, pp. 1-36.

Campesi Giuseppe (2015) Polizia della frontiera. Frontex e la produzione dello spazio europeo, Roma, Deriveapprodi, 201 p.

Cillo Rossana and Pradella Lucia (2016) New Immigrant's Struggle in Italy's Logistic Industry, Comparative European Politics, 16 (1), pp. 67-84.

Curcio Anna (2015) Practicing militant inquiry: Composition, strike and betting in the logistics workers struggles in Italy, Ephemera - theory \& politics in organization, 14 (3), pp. 375-390.

Curcio Anna e Mellino Miguel Angel (2012) La razza al lavoro. Rileggere il razzismo, ripensare I'antirazzismo in Italia, Roma, manifestolibri, 176 p.

Davis Angela (2016) Freedom is a constant Struggle. Ferguson, Palestine and the Foundations of a Movement, Chicago, Haymarket Books, 158 p.

Dines Nick e Rigo Enrica (2017) Lo sfruttamento umanitario del lavoro. Ipotesi e riflessioni di ricerca a partire dal caso delle campagne del mezzogiorno, in Devi Sacchetto e Sandro Chignola, Le reti del valore : migrazioni, produzione e governo della crisi, Roma, DeriveApprodi, pp. 90-107.

Dines Nick and Rigo Enrica (2015) Postcolonial Citizenships and the "Refugeeization" of the Workforce: Migrant Agricultural Labor in the Italian Mezzogiorno, in Sandra Ponzanesi and Gianmarie Colpani Eds., Postcolonial Transitions in Europe: Contexts, Practices and Politics, Lanham, MD, Rowman and International.

Du Bois William E. B. (1935) Black Reconstruction in America, New York, Pree Press.

Du Bois William E. B. (1903) The Souls of Black Folk, essays and sketches, Chicago, A. C. McClurg \& Co.

Etzold B. (2016) Capitalising on Asylum: The Reconfiguration of Refugees'Access to Local Fields of Labour in Germany, in Refugee Revue. Special Focus Labour, 3, pp. 82-102. 
Fanon Frantz (1956) Racisme et culture, Présence africaine, 1-2 (165-166), pp. 77-84.

Fassin Didier (2012) Humanitarian Reason. A Moral History of the Present, Berkeley, University of California Press, $336 \mathrm{p}$.

Fassin Didier et Fassin Éric (2012) De la question sociale à la question raciale : représenter la société française, Paris, La Découverte, 274 p.

Fassin Éric (2017) Racisme d'État (1/2) : un nouveau "front républicain", Mediapart, 27/11/2017.

Foucault Michel (1998) Bisogna difendere la società, Milano, Feltrinelli, 281 p.

Garelli Glenda andTazzioli Martine (2016)The EU Hotspot approach at Lampedusa, openDemocracy, 26/02/2016, [online]. URL: www.opendemocracy.net

Gilmore Ruth Wilson (2007) Golden Gulag: Prisons, Surplus, Crisis and Opposition in Globalizing California, Berkeley, University of California Press, 388 p.

Gilroy Paul (1987) There Ain't No Black in the Union Jack, London, Routledge, $271 \mathrm{p}$.

Goldberg David Theo (2015) Are we all post-racial yet?, London, Polity Press, $199 \mathrm{p}$.

Grappi Giorgio (2016) Logistica, Roma, Ediesse, 268 p.

Hall Stuart (2015) Cultura, razza, potere, Verona, Ombre corte, 214 p.

Hansen Thomas Blom and Stepputat Finn (2005) Sovereign Bodies. Citizens, Migrants and States in the Postcolonial World, Princeton, Princeton University Press, $366 \mathrm{p}$.

Harvey David (2005) A Brief History of Neoliberalism, Oxford, Oxford University Press, $247 \mathrm{p}$.

Harvey David (2003) The New Imperialism, Oxford, Oxford University Press, $253 \mathrm{p}$.

Marchetti Chiara e Pinelli Barbara (2016) Confini d'Europa. Modelli di controllo e inclusioni formali, Milano, Edizioni libreria Cortina di Milano, 200 p.

Maroufi (2017) Precarious Integration: Labour Market Policies for Refugees or Refugee Policies for the German Labour Market?, Refugee Revue. Special Focus Labour, 3, pp. 15-33.

Mbembe Achille (2003) Necropolitics, Public Culture, 15 (1), pp. 11-40.

Mellino Miguel (2012) Cittadinanze postcoloniali. Appartenenze, razza e razzismo in Europa e in Italia, Roma, Carocci, 135 p.

Mezzadra Sandro et Neilson Brett (2014) Confini e frontiere : la moltiplicazione del lavoro nel mondo globale, Bologna, II Mulino, 465 p.

Mezzadra Sandro and Neilson Brett (2013) Border as Method, or the Multiplication of Labour, Durham, Duke University Press, 365 p.

Neocleous Mark and Kastrinou Maria (2016) The EU Hotspot. Police war against the migrant, Radical Philosophy, 200, pp. 3-9. 
Papadopoulos Dimitrios, Stephenson Niamh and Tsianos Vassilis (2008) Escape Routes: Control and Subversion in the 21st Century, London, Pluto Press, 300 p.

Roediger David R. (2010) How Race survived US History: From Settlement and Slavery to the Obama Phenomenon, London, Verso, 254 p.

Saitta Pietro (2015) Resistenze. Pratiche e margini del conflitto nel quotidiano, Verona, Ombre Corte, 116 p.

Taguieff Pierre-André (1988) La force du préjugé : Essai sur le racisme et ses doubles, Paris, Gallimard, 648 p.

Taylor Keeanga-Yamahtta (2016) From \#Blacklivesmatter to Black Liberation, Chicago, Haymarket Books, 270 p.

Tazzioli M. (2016) Biopolitica attraverso la mobilità nel governo militare-umanitario delle migrazioni, in Marchetti Chiara e Pinelli Barbara, Confini d'Europa. Modelli di controllo e inclusioni formali, Milano, Edizioni libreria Cortina di Milano, pp. 37-62.

Wacquant Loic (2006) Punire i poveri : il nuovo governo dell'insicurezza sociale, Roma, DeriveApprodi, $285 \mathrm{p}$.

Wieviorka Michel (1991) L'espace du racisme, Paris, Seuil, 256 p. 